Palavras chave:

Floresta nativa

Floresta Ombrófila Mista

Manejo sustentado

Histórico:

Recebido 05/02/2013

Aceito $21 / 01 / 2015$

Keywords:

Native forest

Mixed Ombrophilous Forest

Sustained management

Correspondência: aebling@hotmail.com

\section{MODELAGEM DE OCORRÊNCIA DE COORTES NA ESTRUTURA DIAMÉTRICA DA Araucaria angustifolia (Bertol.) Kuntze}

RESUMO: Estudos referentes à estrutura diamétrica das florestas nativas são essenciais para o entendimento do desenvolvimento, fornecer parâmetros do crescimento e produção suficientes para gerar estimativas que subsidiem o manejo sustentado. No entanto, a modelagem matemática de funções probabilísticas, como as de densidade, tornam-se de difícil aplicação em distribuições multimodais. A espécie Araucaria angustifolia (Bertol.) Kuntze, de importância social, ambiental e econômica, apresenta padrão de distribuição multimodal, formando unidades demográficas denominadas de coortes, que se originam, em decorrência de agentes antrópicos e naturais que atuam nos nichos. Logo, tomando com base dados inventariados de árvores com diâmetro à altura do peito, igual ou maior que $9,5 \mathrm{~cm}(\mathrm{DAP} \geq 9,5 \mathrm{~cm})$, oriundos da Floresta Nacional de São Francisco de Paula, RS, foram testadas diferentes funções densidade de probabilidade. $O$ melhor ajuste à série de dados consiste em uma função truncada de polinômio de sétimo grau, que, além de manter valores ajustados muito próximos aos observados, manteve a configuração multimodal da distribuição.

\section{MODELING THE OCCURRENCE OF COHORTS IN THE DIAMETRIC STRUCTURE OF Araucaria angustifolia (Bertol.) Kuntze}

ABSTRACT: Studies concerning the diameter structure are essential for understanding the development of native forests, provide growth and yield parameters sufficient to generate estimates that support sustainable management. However, the mathematical modeling of probability functions, such as density, becomes difficult to implement in multi-modal distributions, found in forest species and communities. The species Araucaria angustifolia (Bertol.) Kuntze, of social, environmental and economic features, presents multimodal distribution pattern forming units called demographic cohorts, which arise due to natural and anthropogenic agents that operate in niches. Therefore, taking inventory database of trees with a diameter at breast height equal to or greater than 9.5 $\mathrm{cm}(\mathrm{DBH} \geq 9.5 \mathrm{~cm})$, from the National Forest of São Francisco de Paula, RS, it was possible to test different probability density functions. The best fit to the data series consists of a truncated polynomial function of seventh degree, which besides maintaining fitted values very close to those observed for total and diameter classes, maintained the multimodal distribution characteristic. 


\section{INTRODUÇÃO}

O manejo florestal sustentado consiste na exploração e manutenção dos benefícios econômicos, sociais e ambientais que os ecossistemas dispõem, paralelamente, considerando os mecanismos de sustentação desses ambientes. $\bigcirc$ Brasil detém a segunda maior área florestal do mundo, com 516 milhões de hectares, sendo, desse total, 98,7\% constituídos de florestas naturais (SERVIÇO FLORESTAL BRASILEIRO - SFB, 2010). Logo, torna-se evidente a importância dos estudos que propiciem um maior entendimento dos processos envolvendo as florestas naturais, de modo a favorecer o manejo e continuidade desses recursos. O desenvolvimento alavancado pelo capital oriundo de recursos florestais deve ser conciliado com o reconhecimento da importância de seus benefícios, valorizando o manejo e preservação da cobertura florestal (BRENA, 1996).

A complexidade das interações sinecológicas das florestas heterogêneas multiâneas, dificulta o emprego de modelos matemáticos tradicionalmente empregados. Como exemplo, a distribuição diamétrica da Araucaria angustifolia (Bertol.) Kuntze, que assume distribuições multimodais, diferenciando do modelo de distribuição para florestas balanceadas citado por Meyer et al. ( I 96I), que segue um padrão exponencial negativo. Além disso, a escassez de informações relativas à dinâmica florestal dificulta, ainda mais, estimar valores que propiciem uma exploração racional, sem comprometer a continuidade dos recursos no meio ambiente, e gerar estimativas para definição de ciclos exploratórios (VANCLAY et al., 1997).

Entre as metodologias usualmente utilizadas para prognosticar o volume e efetivar o manejo de florestas heterogêneas, podem ser consideradas a razão de movimentação dos diâmetros, e as cadeias de Markov. Em comum, esses dois modelos são passíveis de refinamentos, citado por Sanquetta et al. (1995), com a aplicação de modelos matemáticos, como as funções densidade de probabilidade, melhorando estimativas. Somado a isso, a partir do contexto de distribuições probabilísticas, funções matemáticas permitem prever fenômenos, favorecendo o estudo do comportamento de variáveis do meio físico ou biológico (SILVA et al., 2003).

Diante do exposto, o presente estudo tem como metas aplicar a modelagem matemática à distribuição de frequências da $A$. angustifolia, selecionar o modelo que melhor se ajuste à distribuição de frequências, permitindo, com isso, gerar estimativas mais condizentes com a configuração da distribuição dos diâmetros entre classes.

\section{MATERIAL E MÉTODOS}

Os dados que subsidiaram as informações do presente estudo são provenientes do Projeto Ecológico de Longa Duração, intitulado: "Conservação e Manejo Sustentável de Ecossistemas Florestais - Bioma Floresta de Araucária e suas Transições”, efetivado na Floresta Nacional de São Francisco de Paula, RS, localizada entre as coordenadas $29^{\circ} 24$ e $29^{\circ} 27^{\prime}$ de latitude Sul e $50^{\circ} 22^{\prime}$ e $50^{\circ} 25^{\prime}$ de longitude Oeste, mesorregião nordeste do estado. A vegetação predominante local é a Floresta Ombrófila Mista ou Floresta com Araucária.

A altitude média do local é de 900 metros acima do nível do mar, com frequente formação de geadas e queda de neve nos meses mais frios (FERNANDES; BACKES, 1998). De acordo com a classificação global dos tipos climáticos desenvolvida por Köppen, o clima da região é do tipo "Cfb”, mesotérmico e superúmido, com verão brando e inverno frio.

A. angustifolia foi à espécie escolhida para desenvolver o estudo, em razão de sua importância ecológica e social na Floresta Ombrófila Mista, além de seu elevado potencial silvicultural, com ampla gama de produtos e utilizações (CARVALHO, 2003). Foram selecionados os indivíduos inseridos na área amostral, correspondente a 10 parcelas com área de I ha $(100 \times 100 \mathrm{~m})$ cada, cujo diâmetro à altura do peito fosse igual ou superior a 9,5 cm (DAP $\geq 9,5)$.

Foram ajustadas funções clássicas no meio florestal: Beta, Exponencial, Gama, Normal (Gauss) e Weibull (3 parâmetros), além das funções multimodais elaboradas por Silva et al. (2003) e Weber (20II). As funções foram ajustadas por meio de regressão não-linear, pelo método dos mínimos quadrados com múltiplas iterações, segundo o procedimento de Marquardt (1963), utilizando o software Table Curve 2 d. Os parâmetros utilizados para apontar o modelo mais acurado foram: coeficiente de determinação ajustado $\left(\mathrm{R}_{\text {ajustado }}^{2}\right)$, erro padrão da estimativa $\left(\mathrm{S}_{\mathrm{yx}} \%\right)$ e teste de Aderência de Kolgomorov-Smirnov (K-S), cujas hipóteses: $\mathrm{H}_{0}$ consiste na aderência entre dados observados e ajustados, em contraponto, a hipótese alternativa $\left(\mathrm{H}_{1}\right)$ pressupõe não aderência.

O modelo selecionado, aplicado na distribuição de frequências observadas ( $\left.\mathrm{N}_{\text {observado }}\right)$, gerou a distribuição ajustada $\left(\mathrm{N}_{\text {ajustado }}\right)$, sendo analisada a distribuição por classes e o total, e, a partir desses, calculada a área basal observada e ajustada, tomando por base o valor central de cada classe diamétrica (Tabela I). 
TABELA 1 Distribuição de frequências e área basal, observada e ajustada para Araucaria angustifolia. Observed and fitted frequency distribution and basal

TABLE 1 area for Araucaria angustifolia.

\begin{tabular}{ccccc}
\hline $\begin{array}{c}\text { Centro } \\
\text { de classe }\end{array}$ & $\mathrm{N}_{\text {observado }}$ & $\mathrm{N}_{\text {ajustado }}$ & $\mathrm{G}_{\text {observado }}$ & $\mathrm{G}_{\text {ajustado }}$ \\
\hline$K_{1}$ & $b_{1,1}$ & $b_{1,2}$ & $\left(\frac{\pi k_{1}^{2}}{4}\right) b_{1,1}$ & $\left(\frac{\pi k_{1}^{2}}{4}\right) b_{1,2}$ \\
$K_{2}$ & $b_{2,1}$ & $b_{2,2}$ & $\left(\frac{\pi k_{2}^{2}}{4}\right) b_{2,1}$ & $\left(\frac{\pi k_{2}^{2}}{4}\right) b_{2,2}$ \\
$K_{3}$ & $b_{3,1}$ & $b_{3,2}$ & $\left(\frac{\pi k_{3}^{2}}{4}\right) b_{3,1}$ & $\left(\frac{\pi k_{3}^{2}}{4}\right) b_{3,2}$ \\
$\vdots$ & $\vdots$ & $\vdots$ & $\vdots$ \\
$K_{n}$ & $b_{n, 1}$ & $b_{n, 2}$ & $\left(\frac{\pi k_{n}^{2}}{4}\right) b_{n, 1}$ & $\left(\frac{\pi k_{n}^{2}}{4}\right) b_{n, 2}$
\end{tabular}

Por meio dos valores observados e ajustados de frequência por classe diamétrica e área basal, foi realizada a análise gráfica, avaliando o paralelismo entre as distribuições.

\section{RESULTADOS E DISCUSSÃO}

Os diâmetros de Araucaria angustifolia foram agrupados em II classes que, apesar de apresentar uma tendência na redução de frequência à medida que ocorre o aumento dos diâmetros, observa-se uma configuração multimodal (Figura I). Configuração semelhante foi observada no estado do Rio Grande do Sul por Pizzato (1999) e Souza (2007) no estado do Paraná, pressupondo uma característica intrínseca da espécie.

Com relação à origem das distribuições multimodais, consideram-se hipóteses, como a

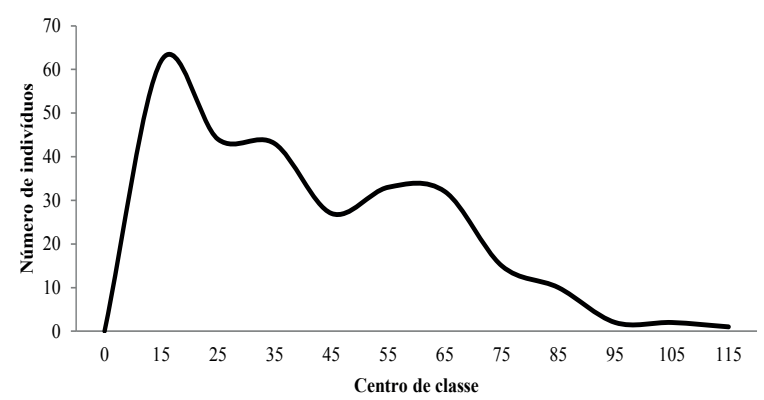

FIGURA 1 Distribuição de frequências em classes diamétricas para Araucaria angustifolia.

FIGURE 1 Frequency distribution in diameter classes for Araucaria angustifolia. formação de unidades demográficas por um grupo de árvores recrutadas em um mesmo intervalo de tempo, constituindo uma coorte. Conforme citado por Ogden (1985), que, analisando o recrutamento de Agathis australis (D. Don), (família Araucariaceae), espécie endêmica da Nova Zelândia, verificou que ela tem regeneração fortemente dependente de distúrbios que favorecem o surgimento de coortes. Como A. angustifolia, a exemplo de Agathis australis, conta com a característica de um longo ciclo de vida, aumentam as probabilidades de ocorrência de distúrbios como incêndios florestais, severas condições climáticas, tempestades, entre outros, que propiciam a formação das coortes (OGDEN; STEWART, (995).

Claessens et al. (2006) definem o início de uma coorte, a partir do momento em que uma maior incidência solar penetra no bosque da floresta, posterior a algum distúrbio, favorecendo a regeneração natural. Concomitante ao desenvolvimento das plântulas ocorre $\circ$ autodesbaste, porém sem perda no valor total de biomassa, sendo este observado no período de seneiscência das árvores. O ciclo se renova à medida que novos distúrbios reincidam sobre a floresta.

Embora a seneiscência promova uma redução de densidade na coorte um número limitado de árvores de grande porte permanecem, podendo novas coortes se estabelecerem, porém, com densidade inferior a coorte matriz, por haver um ambiente mais competitivo na floresta (CLAESSENS et al., 2006). Essa observação pode ser aplicada na distribuição da A. angustifolia, em que a moda das classes superiores representa a primeira geração de uma coorte, estabelecida pela incidência de algum distúrbio na floresta. Já, a moda nas classes inferiores representa a segunda geração da coorte, com maior densidade que a primeira geração, decorrente da menor influência da seneiscência.

Além das coortes, devem-se considerar aspectos como a interferência antrópica. Embora a área de pesquisa não apresente histórico de exploração madeireira, sua região de entorno foi severamente alterada e empobrecida de sua constituição original, essencialmente pela atividade pastoril e exploração madeireira desencadeada a partir do século XVII (BEHLING et al., 2004). Bitencourt e Krauspenhar (2006) salientam a influência da ação antrópica pré-histórica, onde nativos tinham como a base de sua alimentação vegetal a semente de $A$. angustifolia, constituindose de agentes capazes de alterar a paisagem natural e interferir na distribuição natural da espécie, a exemplo da distribuição da castanheira do Pará (Bertholletia excelsa 
Bonpl.), que está fortemente associada a sítios indígenas que realizavam seu plantio (POSEY, 1985; SIMÕES; ARAÚJO-COSTA, 1987).

Portanto, evidencia-se que uma série de agentes antrópicos e naturais, que podem estar atrelados, influenciando na configuração de distribuição multimodal da A. angustifolia. A intensidade com que cada um desses agentes interfere na distribuição dessa espécie se torna de difícil mensuração, em razão da amplitude de tempo e disponibilidade de dados históricos para formalizar uma conclusão. No entanto, independentes dos agentes causadores, se tornam evidentes a presença
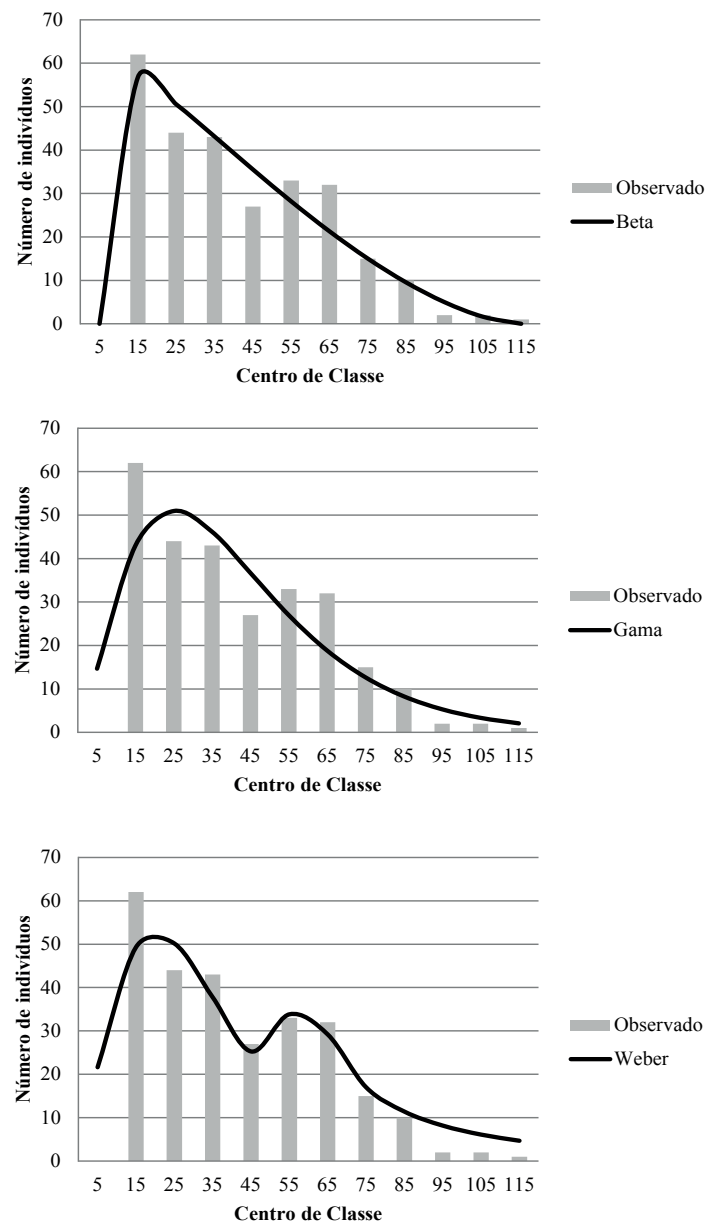

de duas coortes, condicionadas a eventos favoráveis para seu estabelecimento no passado, e que, de certo modo, restringe a aplicação de modelos matemáticos tradicionais.

Vanclay (1994) salienta que embora a modelagem matemática seja de grande valia para gerar estimativas acerca da produção florestal, as características multimodais de distribuição limitam a efetivação dessas. Logo, funções densidade de probabilidade tradicionalmente empregadas no meio florestal não apresentam um ajuste satisfatório na distribuição da $A$. angustifolia (Figura 2).
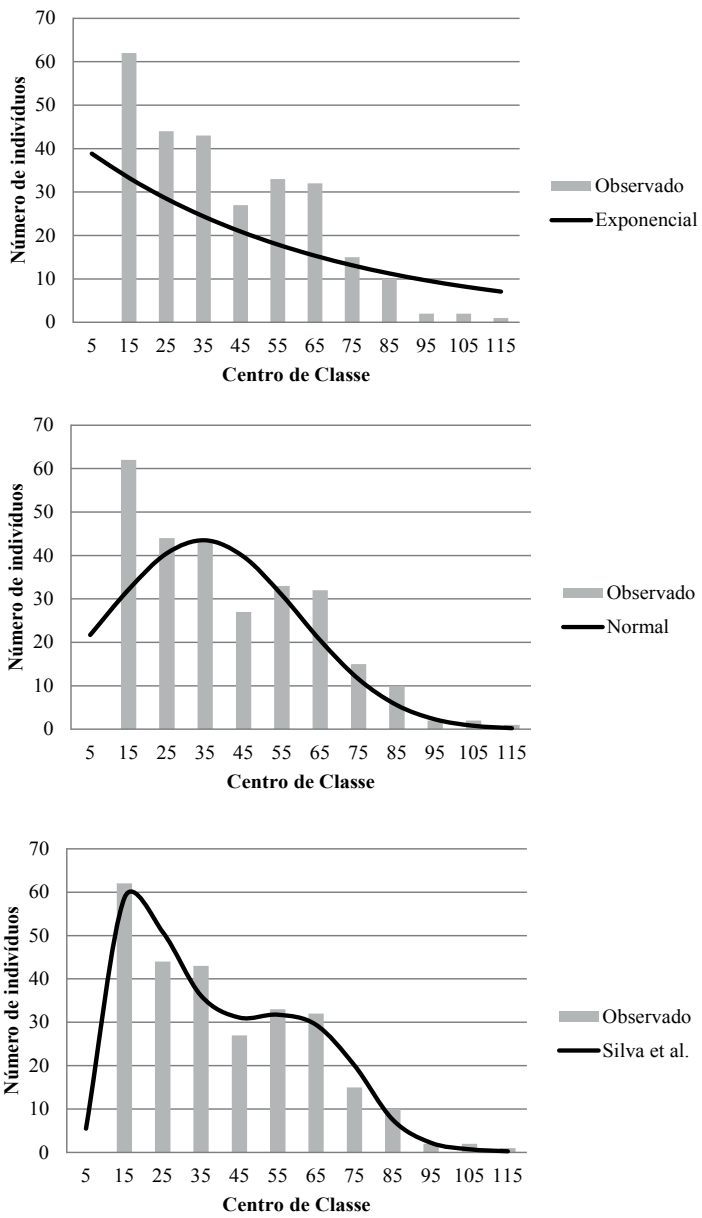

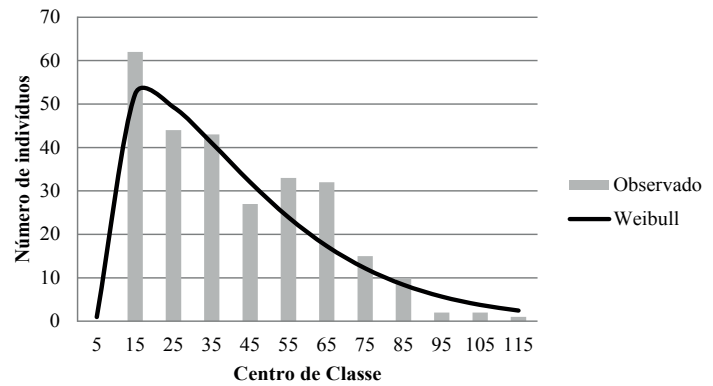

FIGURA 2 Distribuição de frequências observada e ajustada em classes diamétricas para os modelos testados em Araucaria angustifolia. FIGURE 2 Observed and fitted frequency distribution in diameter classes for tested models for Araucaria angustifolia. 
Entre as funções testadas, os modelos ajustados de Silva et al. (2003) e Weber (20II) foram os que apresentaram melhor capacidade de explicar as relações biológicas entre as variáveis, configurando uma distribuição multimodal, porém, o modelo de Weber (20ll) não apresentou aderência, em razão, essencialmente, da dificuldade de seu ajuste na primeira classe diamétrica. Indicaram aderência pelo teste K-S os modelos Beta, Gama, Normal e a função truncada de Silva et al. (2003) (Tabela 2).

TABELA 2 Eficiência das equações de frequência testadas para distribuição de Araucaria angustifolia.

Efficiency of the distribution equations tested for

TABLE 2 frequency of Araucaria angustifolia.

\begin{tabular}{|c|c|c|c|c|c|c|}
\hline & $P$ & $\mathrm{R}_{\text {ajustado }}^{2}$ & $S_{y x} \%$ & $\mathrm{D}_{\text {calc }}$ & $\mathrm{D}_{\text {tab. } 0,5}$ & $\begin{array}{c}\text { Hipótese } \\
\text { Ho }\end{array}$ \\
\hline Beta & 2 & 0,92 & 4,61 & 0,03668 & 0,08261 & $\begin{array}{c}\text { Não } \\
\text { rejeita }\end{array}$ \\
\hline Exponencial & 1 & 15 & 16,20 & 0,23517 & 0,08261 & Rejeita \\
\hline Gama & 2 & 0,75 & 8,37 & 0,05683 & 0,08261 & $\begin{array}{l}\text { Não } \\
\text { rejeita }\end{array}$ \\
\hline Normal & 2 & 0,55 & 11,14 & 0,08017 & 0,08261 & $\begin{array}{l}\text { Não } \\
\text { rejeita }\end{array}$ \\
\hline $\begin{array}{l}\text { Silva et al. } \\
(2003)\end{array}$ & 7 & 0,94 & 3,77 & 0,03291 & 0,08261 & $\begin{array}{l}\text { Não } \\
\text { rejeita }\end{array}$ \\
\hline $\begin{array}{l}\text { Weber } \\
(2011)\end{array}$ & 4 & 0,74 & 7,58 & 0,08709 & 0,08261 & Rejeita \\
\hline Weibull & 3 & 0,86 & 5,97 & 0,10575 & 0,08261 & Rejeita \\
\hline
\end{tabular}

${ }^{*} \mathrm{P}=$ Parâmetro

Os demais critérios seletivos baseados no coeficiente de determinação ajustado e erro padrão da estimativa indicam que a distribuição de Silva et al. (2003), além de apresentar o menor erro padrão entre os modelos testados $\left(\mathrm{S}_{\mathrm{yx}} \%=3,77\right)$, apresentou a maior capacidade de ajuste nas variáveis, observando-se o maior coeficiente de determinação entre os modelos $\left(R_{\text {aiustado }}^{2}=0,94\right)$, sendo, por esse motivo, escolhido como o modelo mais acurado.

O modelo Beta indicou o segundo melhor ajuste, com coeficiente de determinação e erro padrão da estimativa, pouco inferiores ao modelo de melhor ajuste $\left(R_{\text {ajustado }}^{2}=0,92\right.$ e $\left.S_{y x} \%=4,61\right)$. Porém, a função Beta não apresenta plasticidade em sua distribuição de frequências, sendo essa desejada para manter as características ajustadas semelhantes às observadas. $O$ modelo de Weibull, amplamente usado na área florestal, mesmo com bom ajuste de sua equação $\left(R_{\text {ajustado }}^{2}=0,86\right)$ e baixo erro padrão $\left(S_{y x} \%=5,97\right)$, não apresentou aderência às frequências observadas.

A função truncada de Silva et al. (2003), composta de três polinômios $\left(g_{1}, g_{2}\right.$ e $\left.g_{3}\right)$, responsáveis pelo ajuste das classes iniciais, intermediárias e finais, respectivamente, pode ser definida, genericamente, conforme mostrado abaixo, sendo $I_{1}$ e $I_{2}$ os limites das classes onde serão ajustadas respectivamente as funções $c_{1} x^{d}$ e $c_{2} \frac{1}{x^{h}} ; n, d$ e $h$ inteiros psitivos; $a_{l}, a_{2}, a_{3}, a_{m}, c_{1}$ e $c_{2}$ números reais $\mathrm{e} k$ correspondente à integral $\int_{-\infty}^{\infty}\left[c_{1} x^{d}+\left(a_{1} x^{n}+a_{2} x^{n-1}+a_{3} x^{n-2}+\ldots+a_{m}\right)+c_{2} \frac{1}{x^{n}}\right] d x$. $f(x)=\frac{1}{k} \begin{cases}g_{1}=c_{1} x^{d} & \text { se } 0 \leq x<I_{1} \\ g_{2}=a_{1} x^{n}+a_{2} x^{n-1}+a_{3} x^{n-2}+\ldots+a_{m} & \text { se } I_{1} \leq x \leq I_{2} \\ g_{3}=c_{2} \frac{1}{x^{h}} & \text { se } x>I_{2} \\ 0 & \text { e.o.c. }\end{cases}$

Como o ajuste do polinômio de sétimo grau na distribuição de frequências da $A$. angustifolia não era adequado para as extremidades da distribuição de frequências, foram elaboradas as equações $g_{\text {, }}$ para a primeira classe, e $g_{3}$ para as últimas quatro classes diamétricas, considerando que elas constituem uma secção cônica (hipérbole), e de modo que $g_{1}, g_{2}$ e $g_{3}$ configurem uma função contínua e convergente no infinito. Os valores correspondentes de $g_{1}$ e $g_{3}$ foram calculados a partir das ordenadas dos limites de classe $I_{\text {I }}$ e $I_{2}$. Posteriormente, são determinados os valores de $c_{1}$ e $c_{2}$ a partir de seus respectivos pares de ordenadas $\left(I_{1}\right.$ e $\left.I_{2}\right)$, garantindo a continuidade da função. Os expoentes $d$ e $h$, das equações $g_{1}$ e $g_{3}$, foram selecionados com valores que melhor atenderam à tendência dos dados. A função densidade de probabilidade $f(x)$ foi obtida multiplicando $g(x)$ pela integral $k=\int_{-\infty}^{\infty} g(x) d x$.

$$
f(x)=\frac{1}{3515,31143} \begin{cases}g_{1}=0,043925 x^{3} & \text { se } 0 \leq x \\ g_{2}=-89,796841+24,363343 x & <15 \\ -1,433269 x^{2}+0,038231 x^{3}-0,000514 x^{4} & \text { se } 15 \leq x \\ +3,389234 \cdot 10^{-6} x^{5}-8,673488 \cdot 10^{-9} x^{6} & \leq 85 \\ g_{3}=1,26219 \cdot 10^{22} \cdot \frac{1}{x^{11}} & \text { se } x>85 \\ 0 & \text { e.o.c. }\end{cases}
$$

As frequências observadas ( $\left.N_{\text {observado }}\right)$ foram aplicadas na função densidade de probabilidade de Silva et al. (2003), gerando as frequências ajustadas $\left(\mathrm{N}_{\text {ajustado }}\right)$. $\mathrm{O}$ ajuste indicou estimativas de frequência com valor total pouco inferior ao valor observado $\left(0,32\right.$ ind $\cdot$ ha $\left.^{-1}\right)$, assumindo, com isso, também, uma leve redução no valor total de área basal $\left(0,173|| \mathrm{m}^{2} \cdot \mathrm{ha}^{-1}\right)$ (Tabela 3$)$. 
As diferenças encontradas entre os valores observados e ajustados podem ser atribuídas ao erro intrínseco do modelo escolhido, e podem ser considerados pequenos, não interferindo de maneira significativa nos resultados esperados para um manejo florestal adequado da espécie. A análise da distribuição permite ainda inferir que as menores classes diamétricas, embora representem as maiores frequências, não apresentam os valores mais significativos em área basal, sendo esses localizados nas classes diamétricas intermediárias (Figura 3).

TABELA 3 Valores de frequência e área basal, por classe diamétricas e totais, observados ajustados para Araucaria angustifolia.

TABLE 3 Observed and fitted frequency and basal area values by diameter classes and totals for Araucaria angustifolia.

\begin{tabular}{ccccc}
\hline $\begin{array}{c}\text { Centro } \\
\text { de classe } \\
(\mathrm{cm})\end{array}$ & $\begin{array}{c}\mathrm{N}_{\text {observado }} \\
(\mathrm{ha})\end{array}$ & $\begin{array}{c}\mathrm{N}_{\text {ajustado }} \\
(\text { ha })\end{array}$ & $\begin{array}{c}\mathrm{G}_{\text {observado }} \\
\left(\mathrm{m}^{2} \cdot \mathrm{ha}^{-1}\right)\end{array}$ & $\begin{array}{c}\mathrm{G}_{\text {ajustado }} \\
\left(\mathrm{m}^{2} \cdot \mathrm{ha}^{-1}\right)\end{array}$ \\
\hline 15 & 6,2 & 5,86 & 0,10956 & 0,10359 \\
25 & 4,4 & 5,08 & 0,21598 & 0,24941 \\
35 & 4,3 & 3,61 & 0,41371 & 0,34758 \\
45 & 2,7 & 3,11 & 0,42942 & 0,49445 \\
55 & 3,3 & 3,18 & 0,78402 & 0,75444 \\
65 & 3,2 & 2,94 & 1,06186 & 0,97640 \\
75 & 1,5 & 2,01 & 0,66268 & 0,88715 \\
85 & 1,0 & 0,75 & 0,56745 & 0,42800 \\
95 & 0,2 & 0,22 & 0,14176 & 0,15729 \\
105 & 0,2 & 0,07 & 0,17318 & 0,06390 \\
115 & 0,1 & 0,03 & 0,10387 & 0,02818 \\
\hline Total & 27,1 & 27,42 & 4,66350 & 4,49039 \\
\hline
\end{tabular}

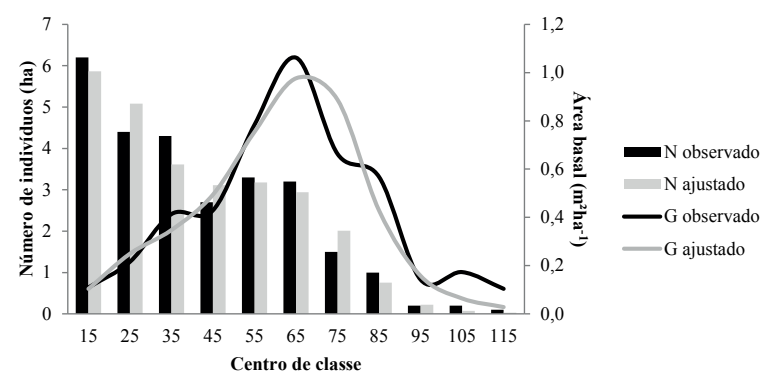

FIGURA 3 Distribuição diamétrica e área basal, observadas e ajustadas por classes para Araucaria angustifolia.

FIGURE 3 Observed and fitted diametric distribution and basal area by classes for Araucaria angustifolia.
A curva de área basal ajustada ( $\left.G_{\text {ajustado }}\right)$ manteve simetria com a observada $\left(\mathrm{G}_{\text {observado }}\right)$, porém, propiciando uma maior homogeneidade ou "alisamento" na distribuição, que pode favorecer, por exemplo, a efetivação de prognoses, essencialmente aquelas aplicadas para maiores amplitudes de tempo, reduzindo, com isso, valores discrepantes.

\section{CONCLUSÕES}

As distribuições multimodais, como a encontrada para $A$. angustifolia, são características do ciclo de coortes, e correspondem aos avanços intrínsecos condicionados ao nicho. Ações antrópicas podem ter influência nas distribuições, interferindo, tanto com uma silvicultura rudimentar pelos povos nativos, ou pela exploração dos produtos madeiráveis e não madeiráveis;

Funções densidade de probabilidade, usualmente empregadas no meio florestal, não apresentam um bom resultado frente à modelagem matemática em distribuições multimodais, como no caso de $A$. angustifolia, sendo a função polinomial truncada de Silva et al. (2003) o modelo que melhor se ajusta a esse tipo de distribuição. Logo, essa função apresenta-se como uma alternativa para distribuições multimodais, podendo ser empregada para demais espécies ou comunidades florestais, desde que apresente um ajuste satisfatório.

\section{REFERÊNCIAS}

BEHLING, H.; PILLAR, V. P.; ORLÓCI, L.; BAUERMANN, S. G. Late quaternary Araucaria forest, grassland (campos), fire and climate dynamics, studied by high-resolution pollen, charcoal and multivariate analysis of the Cambará do Sul core in southern Brazil. Palaeogeography, Palaeoclimatology, Palaeoecology, Davis, v. 203, p. 277297, 2004.

BITENCOURT, A. L. V.; KRAUSPENHAR, P. M. Possible prehistoric antropogenic effect on Araucaria angustifolia (Bert.) O. Kuntze expansion during the late holocene. Revista Brasileira de Paleontologia, Porto Alegre, v. 9, n. I, p. 15-26, jan./abr. 2006.

BRENA, D. Proposição de um sistema de inventário florestal nacional para o Brasil. Ciência Florestal, Santa Maria, v. 6, n. I, p. 109-127, 1996.

CARVALHO, P. E. R. Espécies arbóreas brasileiras. Brasília: EMBRAPA Informação Tecnológica, 2003. 1039 p. 
CLAESSENS, L.; VERBURG, P. H.;SCHOORL,J. M.; VELDKAMP, A. Contribution of topographically based landslide hazard modelling to the analysis of the spatial distribution and ecology of Kauri (Agathis australis). Landscape Ecology, Dordrecht, v. 21, p. 63-76, 2006.

FERNANDES, A. V.; BACKES, A. Produtividade primária em floresta com Araucaria Angustifolia no Rio Grande do Sul. Iheringia Série Botânica, Porto Alegre, v. 5I, n. I, p. 63$78,1998$.

MARQUARDT, D. W. An algorithm for least squares estimation of nonlinear parameters. Journal of the Society for Industrial and Applied Mathematics, Auckland, v. I I, n. 2, p. 43I-44I, 1963.

MEYER, H. A.; RECKNAGEL, A. B.; STEVENSON, D. D.; BARTOO, R. A. Forest management. $2^{\text {nd }}$ ed. New York: Ronald, I96I. 282 p.

OGDEN, J. An introduction to plant demography with special reference to New Zealand trees. New Zealand Journal of Botany, Wellington, v. 23, p. 75I-772, 1985.

OGDEN, J.; STEWART, G. H. Community dynamics of New Zealand conifers. In: ENRIGHT, N. J.; HILL, R. S. (Ed.). Ecology of the Southern Conifers. Melbourne: Melbourne University, 1995. p. 8I-II9.

PIZATTO, W. Avaliação biométrica da estrutura e da dinâmica de uma Floresta Ombrófila Mista em São João do Triunfo, PR: 1995 a 1998. 1999. I72 f. Dissertação (Mestrado em Ciências Florestais) - Universidade Federal do Paraná, Curitiba, 1999.

POSEY, D. Indigenous menagement of tropical forest ecosystems: the case of kayapo indians of the Brazilian Amazon. Agroforestry Systems, Amsterdam, v. 3, p. I39-I58, 1985.
SANQUETTA, C. R.; ANGELO, H.; BRENA, D. A.; MENDES, J. B. Predição da distribuição diamétrica, mortalidade e recrutamento de floresta natural com matriz Markoviana de potência. Floresta, Curitiba, v. 24, n. I, p. 23-36, 1995.

SERVIÇO FLORESTAL BRASILEIRO. Florestas do Brasil em resumo 20 I 0: dados de 2005-20 I0. Brasília, 2010. $152 \mathrm{p}$.

SILVA, E. Q. da; PÉLLICO NETTO, S.; MACHADO, S. do; SANQUETTA, C. R. Função de densidade de probabilidade aplicável à ciência florestal. Revista Floresta, Curitiba, v. 33, n. 3, p. 285-294, 2003.

SIMÕES, M.; ARAÚJO-COSTA, F. Pesquisas arqueológicas no baixo rio Tocantins. Revista de Arqueologia, Belém, v. 4, n. I, p. II-28, 1987.

SOUZA, A. F. Ecological interpretation of multiple population size structures in trees: the case of Araucaria angustifolia in South America. Austral Ecology, Malden, v. 32, p. 524533, 2007.

VANCLAY, J. K. Modelling forest growth and yield: applications to mixed tropical forests. Wallingford: $C A B$ International, 1994. 330 p.

VANCLAY, J. K.; GILLICON, A. N.; KEENAN, R. J. Using functional attributes to quantify site productivity and growth patterns in mixed forests. Forest Ecology and Management, Amsterdam, v. 94, p. 149-163, 1997.

WEBER, S. H. Desenvolvimento de uma função densidade de probabilidade multimodal aplicável à ciência florestal. 20II. I32 f. Tese (Doutorado em Manejo Florestal) - Universidade Federal do Paraná, Curitiba, $201 \mathrm{I}$. 
